\title{
STRUCTURAL DAMPING MODELS FOR PASSIVE AEROELASTIC CONTROL
}

\author{
Marco Eugeni ${ }^{1}$, Francesco Saltari $^{1}$, Franco Mastroddi ${ }^{1}$, Cristina Riso $^{2}$ \\ ${ }^{1}$ Sapienza University of Rome \\ Via Eudossiana 18, 00184 Rome, Italy \\ marco.eugeni@uniroma1.it \\ francesco.saltari@uniroma1.it \\ franco.mastroddi@uniroma1.it \\ ${ }^{2}$ University of Michigan \\ 1320 Beal Avenue, 48109 Ann Arbor, Michigan USA \\ criso@umich.edu
}

Keywords: damping modeling, passive flutter suppression, viscoelastic materials

\begin{abstract}
Aeroelastic qualification requirements are typically met by sizing aircraft to achieve adequate stability margins and keep peak gust responses below specified thresholds. A possible alternative approach is delaying flutter and alleviating gust response by embedding dissipative materials in structural components. This approach requires accurate damping models applicable to analyze complex configurations. This paper compares three damping models suitable for finite element aeroelastic analysis: the viscous model, the hysteretic model, and a generalized Biot model previously proposed by the authors. The damping models are applied to the flutter suppression and gust load alleviation of a practical aeroelastic testbed using dissipative skin patches. Results obtained using different damping models are compared to provide modeling recommendations for passive flutter suppression and gust alleviation studies.
\end{abstract}

\section{INTRODUCTION}

A correct modeling of damping properties of viscoelastic materials is a key point in modern aerospace applications. Indeed, designing damping devices for suppressing undesirable aeroelastic vibrations requires damping models that accurately capture material dissipation.

The linear theory of viscoelastic materials was initially formulated by Boltzman in 1874 [1]. Later, Noll and Coleman [2-5] introduced the concept of fading memory and modeled the nonconservative portion of the stress tensor using fading memory functions. A honest and deep critic of the concept of fading memory and its implications in the mathematical properties of the viscoelastic operator can be found in Refs. [6,7]. For a complete overview on the modeling of viscoelastic continua, the reader can refer to Refs. [2, 4,5,8].

From a practical point of view, damping models must be readily applicable to large-scale finite element models for analyzing practical configurations. Furthermore, they need to be characterized by a set of parameters that can easily measured experimentally $[8-11]$. These requirements make the modeling of damping properties in the frequency domain particularly appealing, although it implies a careful mathematical treatment as it will be discussed later. 
Two damping descriptions are widely used in linear finite element analysis [12]: viscous and hysteretic (or structural). The viscous damping model introduces a dissipation term proportional to velocity in the time domain. In a mechanical system described by one degree-of-freedom (DOF) $x$, this term is given by $c \dot{x}$ where $c$ is the viscous damping coefficient. The viscous model is very simple and readily applicable to both frequency-domain and transient analyses. However, it implies that the energy dissipated by the material per harmonic motion cycle varies linearly with frequency. In contrast, experimental evidence shows that the energy dissipated by certain materials (such as metallic materials) per harmonic motion cycle is practically constant in frequency.

For a 1DOF mechanical system, the hysteretic (or structural) damping model introduces a dissipative term of the form $j \eta k \tilde{x}$ in the frequency domain, where the tilde denotes Fourier transforms, $j$ is the imaginary unit, $\eta$ a frequency-constant structural damping coefficient and $k$ the stiffness associated to the degree of freedom $x$. While this frequency-domain description is more physically accurate than the viscous description for a class of materials, it leads to a frequency response function (FRF) with even imaginary part. Moreover, the FRF real and imaginary part do not form a Hilber part, so leading to a non-causal impulse response function (IRF) [9]. An unphysical time-domain system response follows that is complex and non-causal.

Different damping models are available that overcome the above issues while preserving agreement with experimental evidence. One possibility is to use fractional time derivatives where damping parameters are evaluated by directly fitting experimental data [13-16]. Alternatively, a previous work by the authors generalized the 1DOF model by Biot [17] to large-scale finite element models [9]. The generalized Biot model includes a Hilbert self-correction for giving a real and causal time-domain system response. Model parameters can be tuned on experimental data. This model gives a frequency-domain damping description equivalent to a three-parameter Kelvin-Voigt fractional-derivative model [18] and can be readily implemented in off-the-shelf finite element solvers typically used in industrial structural and aeroelastic analyses [9].

The correct modeling of damping in aeroelastic system its critical to their design. Indeed, damping modeling influences stability as well as their post-critical nonlinear dynamic behavior [19, 20]. The scope of this paper is to compare the viscous, structural, and generalized Biot model as applied to an existing aeroelastic testbed [21,22] for body-freedom flutter suppression and gust response alleviation using dissipative skin patches. Results obtained with the different models are compared to provide modeling recommendations for passive flutter suppression and gust response alleviation studies.

The paper is organized as follows. Section 2 summarizes the derivation of the viscous, hysteretic, and generalized Biot damping models from a general first-principle-based viscoelastic formulation, which was detailed in Ref. [9]. A dissipation metric is also introduced to compare the damping models, which are integrated into a linear aeroelastic model for studying their influence on flutter and gust response. Section 3 describes the test case used for the numerical results of Sec. 4. A section of concluding remarks wraps up the paper.

\section{THEORETICAL FORMULATION}

This section recalls the theoretical background on damping modeling for finite element linear structural and aeroelastic analysis. The viscous, hysteretic, and generalized Biot damping models are derived as special cases of a general linear viscoelastic formulation. A dissipation metric is also introduced to compare the damping effectiveness of the models as a function of 
frequency that is later used in the aeroelastic studies.

\subsection{Linear Viscoelastic Damping Modeling}

The stress tensor $\mathbf{T}$ of a solid material can be written as

$$
\mathbf{T}:=\mathbf{T}_{E}+\mathbf{T}_{v i s} \quad \mathbf{T}_{E}:=\left.\rho \frac{\partial e}{\partial \mathbf{E}}\right|_{S=c o n s t}
$$

where $\mathbf{T}_{E}$ is the elastic term and $\mathbf{T}_{v i s}$ the dissipative (viscous) term, $\rho$ is the material density, and $e=e(S ; \mathbf{E})$ the internal energy that is function of the entropy per unit mass $S$ and of the strain tensor $\mathbf{E}$.

The elastic term $\mathbf{T}_{E}$ is a function of the strain tensor only through the constitutive law $\mathbf{T}_{E}=$ $\mathbf{T}_{E}(\mathbf{E})$. This means that the elastic stress depends only on the present strain state, as well known for conservative processes. The dissipative term depends on the strain rate history through

$$
\mathbf{T}_{v i s}(t)=\int_{0}^{+\infty} \boldsymbol{\Phi}(\tau) \dot{\mathbf{E}}(t-\tau) d \tau=\boldsymbol{\Phi}(0) \mathbf{E}(t)+\int_{0}^{+\infty} \dot{\boldsymbol{\Phi}}(\tau) \mathbf{E}(t-\tau) d \tau
$$

where $\dot{\mathbf{E}}$ is the strain-rate tensor, the dot denotes time derivatives, and $\mathbf{\Phi}(\tau)$ is a stress relaxation fourth-order tensor that verifies

$$
\lim _{\tau \rightarrow 0} \Phi(\tau)=0
$$

along with the causality condition $\boldsymbol{\Phi}(\tau)=0$ for $\tau<0$.

Assuming small displacements, the strain and strain rate tensors are written as

$$
\mathbf{E}=\operatorname{sym}(\nabla \mathbf{u}) \quad \dot{\mathbf{E}}=\operatorname{sym}(\nabla \dot{\mathbf{u}})
$$

where $\mathbf{u}$ is the displacement vector. Using a finite element method (FEM) space discretization, this can be written as

$$
\mathbf{u}(\mathbf{x} ; t) \cong \sum_{i=1}^{N} q_{i}(t) \phi^{(i)}(\mathbf{x})
$$

where $\phi^{(i)}$ and $q_{i}$ are the shape function and the nodal displacement (translation or rotation) of the $i$ th FEM node $(i=1, \ldots, N)$. Assuming that the principal directions of $\mathbf{T}_{E}$ and $\mathbf{T}_{v i s}$ keep parallel at any time [10], the stress relaxation tensor can be written as

$$
\dot{\Phi}(\tau)=\eta(\tau) \mathbf{C} \quad \text { with }
$$

where the tensor $\mathbf{C}$ gives the linearly elastic constitutive relation as $\mathbf{T}_{E}=\mathbf{C E}$ and $\eta(\tau)$ is a scalar function that describes the material memory and thus satisfies the causality condition

$$
\eta(\tau)=0 \quad \text { for } \quad \tau<0
$$

With some manipulations [9], the FEM equations for a viscoelastic structure can be cast as

$$
\mathrm{M} \ddot{\mathbf{q}}(t)+\mathrm{Kq}(t)+\sum_{i=1}^{N_{e}} \mathrm{~K}_{e_{i}} \int_{0}^{+\infty} \eta_{e_{i}}(\tau) \mathrm{q}(t-\tau) d \tau=\mathbf{f}(t)
$$


where $\mathrm{q}$ is the $N \times 1$ vector of FEM nodal displacements and rotations, $\mathrm{f}$ is the $N \times 1$ vector of FEM nodal forces and moments, and the $N \times N$ model mass and stiffness matrices are

$$
\mathrm{M}=\sum_{i=1}^{N_{e}} \mathrm{M}_{e_{i}} \quad \mathrm{~K}=\sum_{i=1}^{N_{e}} \mathrm{~K}_{e_{i}}
$$

where $\mathrm{M}_{e_{i}}$ and $\mathrm{K}_{e_{i}}$ are the $N \times N$ contributions from the $i$ th element.

Applying the Borel theorem and the Fourier transform to the time-domain model in Eq. (8) and assuming zero initial conditions, one obtains the frequency-domain representation

$$
\tilde{\mathbf{q}}(\omega)=\mathbf{H}(\omega) \tilde{\mathbf{f}}(\omega)
$$

where $\omega$ is the Fourier variable and the FRF system (10) is given by

$$
\mathrm{H}(\omega):=\left\{-\omega^{2} \mathrm{M}+\sum_{i=1}^{N_{e}} \mathrm{~K}_{e_{i}}\left[1+\tilde{\eta}_{e_{i}}(\omega)\right]\right\}^{-1}
$$

The element dissipation is described by the function

$$
\tilde{\eta}_{e_{i}}(\omega):=\mathfrak{F}\left[\eta_{e_{i}}(\tau)\right]=\mathcal{R}_{e_{i}}(\omega)+j \mathcal{X}_{e_{i}}(\omega)
$$

where $\mathfrak{F}(\bullet)$ denotes the Fourier transform operator. Damping models are typically formulated in the frequency-domain, that is, by assigning $\tilde{\eta}_{e_{i}}(\omega)$, because $\eta_{e_{i}}(\tau)$ cannot be measured experimentally. For being consistent with Eq. (7), the specified $\tilde{\eta}_{e_{i}}(\omega)$ must be such that the real and imaginary part $\mathcal{R}_{e_{i}}(\omega)$ and $\mathcal{X}_{e_{i}}(\omega)$ form a Hilbert pair [23, 24]. This ensures that the inverse Fourier transform is a real and causal time-domain signal.

The viscous damping model is obtained by specializing the FRF (11) to the case

$$
\tilde{\eta}_{e_{i}}(\omega)=2 j \zeta_{e_{i}} \omega / \bar{\omega}
$$

where $\zeta_{e_{i}}$ is a frequency-constant damping coefficient for the $i$ th element and $\bar{\omega}$ is a reference natural frequency. This leads to the FRF

$$
\mathrm{H}_{V}(\omega)=\left\{-\omega^{2} \mathrm{M}+j \omega \mathrm{D}+\mathrm{K}\right\}^{-1}
$$

where the viscous damping matrix is given by

$$
\mathrm{D}=\frac{2}{\bar{\omega}} \sum_{i=1}^{N_{e}} \mathrm{~K}_{e_{i}} \zeta_{e_{i}}
$$

This model gives a real and causal time-domain response, but dissipation varies linearly with frequency which does not agree with experimental evidence for most of materials. In fact, damping effects are underestimated for $\omega<\bar{\omega}$ while they are overestimated for $\omega>\bar{\omega}$.

The hysteretic damping model can be obtained by specializing the FRF (11) to the case

$$
\tilde{\eta}_{e_{i}}(\omega)=j \bar{\eta}_{e_{i}} \operatorname{sgn}(\omega)
$$


where $\bar{\eta}_{e_{i}}$ is an assigned characterizing constant (typically known as loss factor) and the sign function is introduced for ensuring a real time-domain response. This gives the FRF

$$
\mathrm{H}_{H}(\omega)=\left\{-\omega^{2} \mathrm{M}+\sum_{i=1}^{N_{e}} \mathrm{~K}_{e_{i}}\left[1+j \bar{\eta}_{e_{i}} \operatorname{sgn}(\omega)\right]\right\}^{-1}
$$

which does not verify the causality condition because the real and imaginary part of $\tilde{\eta}_{e_{i}}(\omega)$ do not form a Hilbert pair. However, the hysteretic description is very popular in vibration analysis because material loss factors can be measured experimentally and, for certain materials, they are practically constant in frequency.

Finally, the Biot damping model can be obtained by specializing the FRF (11) to the case

$$
\tilde{\eta}_{e_{i}}(\omega)=\frac{2}{\pi} \hat{\eta}_{e_{i}}\left[\ln \sqrt{1+\left(\omega / \epsilon_{e_{i}}\right)^{2}}+j \arctan \left(\omega / \epsilon_{e_{i}}\right)\right]
$$

where the real and imaginary parts form a Hilbert pair, $\hat{\eta}_{e_{i}}$ is a frequency-constant reference loss factor for the $i$ th element, and $\epsilon_{e_{i}}$ a frequency scaling parameter depending on the characteristic relaxation time of the element material. This gives the FRF [9]

$$
\mathrm{H}_{B}(\omega)=\left\{-\omega^{2} \mathrm{M}+\sum_{i=1}^{N_{e}} \mathrm{~K}_{e_{i}}\left[1+\frac{2}{\pi} \hat{\eta}_{e_{i}} \ln \sqrt{1+\left(\frac{\omega}{\epsilon_{e_{i}}}\right)^{2}}+j \frac{2}{\pi} \hat{\eta}_{e_{i}} \arctan \left(\frac{\omega}{\epsilon_{e_{i}}}\right)\right]\right\}^{-1}
$$

that is consistent with Eq. (8) because it ensures a real and causal time-domain system. As for the hysteretic model, the parameters in Eq. (18) can be evaluated based on frequency-domain experimental data [9].

\subsection{Dissipation Metric}

A dissipation metric is introduced for comparing the damping effect of the previously recalled models as a function of frequency. The structure is assumed to undergo a fully developed harmonic motion

$$
\mathbf{u}(\mathbf{x} ; t)=\mathbf{f}(\mathbf{x}) \sin (\omega t)
$$

where $\mathbf{x}$ is position, $\mathbf{f}(\mathbf{x})$ is a displacement field that satisfies the boundary conditions, $\omega$ is the oscillation angular frequency, and $T=2 \pi / \omega$ the corresponding time period. The strain and strain rate fields due to the motion (20) are given by

$$
\mathbf{E}(\mathbf{x} ; t)=\operatorname{sym} \nabla \mathbf{f}(\mathbf{x}) \sin (\omega t) \quad \dot{\mathbf{E}}(\mathbf{x} ; t)=\operatorname{sym} \nabla \mathbf{f}(\mathbf{x}) \omega \cos (\omega t)
$$

The performance of the damping models introduced in Sec.2.1 is compared by quantifying the energy dissipated by the structure over one oscillation period. For the sake of simplicity, the metric is derived below by assuming that the structure is homogeneous. The general case where parts of the structure are associated to different material properties can be treated by assuming a FEM discretization and by summing up contributions for the individual elements. 
The energy dissipated by the structure over one oscillation cycle can be written as

$$
\begin{aligned}
\mathcal{E}_{D}(\omega) & :=\iiint_{\mathcal{V}} \int_{0}^{T} \mathbf{T}_{v i s}: \dot{\mathbf{E}} d t d \mathcal{V} \\
& =\omega \iiint_{\mathcal{V}} \int_{0}^{T} \mathbf{T}_{v i s}: \operatorname{sym} \nabla \mathbf{f} \cos (\omega t) d t d \mathcal{V} \\
& =\omega \iiint_{\mathcal{V}} \int_{0}^{T}\left[\int_{0}^{+\infty} \eta(\tau) \mathbf{C E}(t-\tau) d \tau\right]: \operatorname{sym} \nabla \mathbf{f} \cos (\omega t) d t d \mathcal{V} \\
& =\omega \int_{0}^{T} \int_{0}^{\infty} \eta(\tau) \sin [\omega(t-\tau)] d \tau \cos (\omega t) d t \iiint_{\mathcal{V}} \operatorname{sym} \nabla \mathbf{f} \mathbf{C} \operatorname{sym} \nabla \mathbf{f} d \mathcal{V} \\
& =\mathcal{F}_{\eta}(\omega) \mathcal{F}_{\mathcal{B}}
\end{aligned}
$$

where

$$
\mathcal{F}_{\eta}(\omega):=\omega \int_{0}^{T} \int_{0}^{\infty} \eta(\tau) \sin [\omega(t-\tau)] d \tau \cos (\omega t) d t
$$

depends only on the material dissipation properties while

$$
\mathcal{F}_{\mathcal{B}}:=\iiint_{\mathcal{V}} \operatorname{sym} \nabla \mathbf{f} \mathbf{C} \operatorname{sym} \nabla \mathbf{f} d \mathcal{V}
$$

is related to the maximum elastic energy that can be stored in the structure due to the displacement field $\mathbf{u}$. In fact, assuming a FEM discretization of the displacement field one obtains

$$
\mathcal{F}_{\mathcal{B}}=\sum_{n, m=1}^{N} q_{n} q_{m} \iiint_{\mathcal{V}} \operatorname{sym} \nabla \boldsymbol{\Phi}^{(n)} \mathbf{C} \operatorname{sym} \nabla \boldsymbol{\Phi}^{(n)} d \mathcal{V}=\mathrm{q}^{T} \mathrm{Kq}
$$

where $\mathrm{q}^{T} \mathrm{Kq}$ is twice the elastic energy of the space-discretized structure associated to the assigned displacement field $\mathbf{f}(\mathbf{x})$.

Using the Borel theorem, Eq. 24 can be rewritten in terms of $\tilde{\eta}(\omega)$ as follows:

$$
\begin{aligned}
\mathcal{F}_{\eta}(\omega) & =\omega \int_{0}^{T} \mathfrak{F}^{-1}\left\{\tilde{\eta}(\bar{\omega}) \frac{\pi}{j}[\delta(\bar{\omega}-\omega)-\delta(\bar{\omega}+\omega)]\right\} \cos (\omega t) d t \\
& =\omega \int_{0}^{T} \frac{1}{2 \pi} \int_{-\infty}^{+\infty} \tilde{\eta}(\bar{\omega}) \frac{\pi}{j}[\delta(\bar{\omega}-\omega)-\delta(\bar{\omega}+\omega)] e^{j \bar{\omega} t} d \bar{\omega} \cos (\omega t) d t \\
& =\omega \int_{0}^{T} \frac{\tilde{\eta}(\omega) e^{j \omega t}-\tilde{\eta}(-\omega) e^{-j \omega t}}{2 j} \cos (\omega t) d t \\
& =\omega \int_{0}^{T}|\tilde{\eta}(\omega)| \frac{e^{j\left[\omega t+\varphi_{\tilde{\eta}}(\omega)\right]}-e^{-j\left[\omega t+\varphi_{\tilde{\eta}}(\omega)\right]}}{2 j} \cos (\omega t) d t \\
& =\omega \int_{0}^{T}|\tilde{\eta}(\omega)| \sin \left[\omega t+\varphi_{\tilde{\eta}}(\omega)\right] \cos (\omega t) d t \\
& =\pi|\tilde{\eta}(\omega)| \sin \left[\varphi_{\tilde{\eta}}(\omega)\right]=\pi \operatorname{Im}[\tilde{\eta}(\omega)]
\end{aligned}
$$

where $\mathfrak{F}^{-1}(\bullet)$ denotes the inverse Fourier transform operator and $|\tilde{\eta}(\omega)|$ and $\varphi_{\tilde{\eta}}(\omega)$ are the magnitude and phase of $\eta(\omega)$, respectively. 
Equation (27) can be specialized to the damping models described in Secs. 2.1. For the viscous damping model (13) one has

$$
\mathcal{E}_{D_{V}}(\omega)=2 \pi \zeta \frac{\omega}{\bar{\omega}} \mathcal{F}_{\mathcal{B}}
$$

For the hysteretic damping model $(16)$ one has

$$
\mathcal{E}_{D_{H}}(\omega)=\pi \bar{\eta}_{H} \mathcal{F}_{\mathcal{B}}
$$

where $\bar{\eta}_{H}$ is the hysteretic model loss factor assumed as constant for the entire structure. Finally, for the Biot model (18) one has

$$
\mathcal{E}_{D_{B}}(\omega)=\frac{2}{\pi} \bar{\eta}_{B} \arctan (\omega / \varepsilon) \mathcal{F}_{\mathcal{B}}
$$

where $\eta_{B}$ and $\varepsilon$ are the Biot model reference loss factor and the frequency scaling parameter assumed as constant for the entire structure.

\subsection{Aeroviscoelastic model}

For aeroelastic analyses, the viscoelastic formulation of Sec.2 is combined with a linear doubletlattice method (DLM) unsteady aerodynamic model, leading to the aeroviscoelastic system

$$
\left[s^{2} \overline{\mathrm{M}}+\overline{\mathrm{K}}+\sum_{i=1}^{N_{e}} \tilde{\eta}_{e_{i}}(s) \overline{\mathrm{K}}_{e_{i}}\right] \tilde{\overline{\mathrm{q}}}(s)=\tilde{\overline{\mathrm{e}}}(s)+\tilde{\overline{\mathrm{e}}}_{g}(s)
$$

where $s$ is the Laplace variable, $\tilde{\bar{q}}$ is the vector of the Laplace transforms of the $N_{m}$ modal coordinates $\left(N_{m} \ll N\right), Z$ is the $N \times N_{m}$ matrix of the associated structural eigenvectors, $\overline{\mathrm{M}}=\mathrm{Z}^{\top} \mathrm{MZ}$ is the $N_{m} \times N_{m}$ diagonal modal mass matrix, $\overline{\mathrm{K}}=\mathrm{Z}^{\top} \mathrm{KZ}$ the $N_{m} \times N_{m}$ diagonal modal stiffness matrix, $\overline{\mathrm{K}}_{e_{i}}=\mathrm{Z}^{\top} \mathrm{K}_{e_{i}} \mathrm{Z}$ is the $N_{m} \times N_{m}$ contribution to the modal stiffness matrix from the $i$ th elemental stiffness matrix, $\tilde{\eta}_{e_{i}}(s)$ is the analytical continuation [25] of the function $\tilde{\eta}_{e_{i}}(\omega)$ obtained by replacing $j \omega$ with $s$, $\tilde{\bar{e}}$ is the vector of the $N_{m}$ generalized aerodynamic forces due to the motion of the structure (aeroelastic feedback), and $\tilde{\bar{e}}_{g}$ is the vector of the $N_{m}$ generalized aerodynamic forces due to external gusts. The aeroviscoelastic model (31) can be specialized to the viscous, hysteretic, or Biot damping formulations by specifying the function $\tilde{\eta}_{e_{i}}$ for the model FEM elements $(i=1, \ldots, N)$.

The generalized aerodynamic loads due to the structural motion and gusts are written as

$$
\begin{aligned}
\tilde{\tilde{\mathrm{e}}} & =q_{D} \overline{\mathrm{E}}\left(M_{\infty}, p\right) \tilde{\overline{\mathrm{q}}} \\
\tilde{\overline{\mathrm{e}}}_{g} & =q_{D} \overline{\mathrm{E}}_{g}\left(M_{\infty}, p\right) \tilde{w}_{g}(s)
\end{aligned}
$$

where $q_{D}=\rho_{\infty} U_{\infty}^{2} / 2$ is the freestream dynamic pressure, $\rho_{\infty}$ and $U_{\infty}$ being the freestream density and flight speed, $\overline{\mathrm{E}}$ is the $N_{m} \times N_{m}$ generalized aerodynamic force matrix (GAF), $\overline{\mathrm{E}}_{g}$ is the $N_{m} \times 1$ generalized gust response vector, and $\tilde{w}_{g}(s)$ is the assigned input gust (constant for the entire structure). The linear frequency-domain operators $\bar{E}$ and $\bar{E}_{g}$ are functions of the freestream Mach number $M_{\infty}$ and of the nondimensional Laplace variable $p:=s b / U_{\infty}$, where $b$ is the reference half-chord. These operators are evaluated as a function of the reduced frequency $k=\operatorname{Im}(p)=\omega b / U_{\infty}$, that is, on the imaginary axis of the complex plane. Their validity is then extended to the nondimensional Laplace domain via analytic continuation [25]. 
Substituting Eq. (32) into Eq. (31) one obtains

$$
\left[\frac{p^{2} U_{\infty}^{2}}{b^{2}} \overline{\mathrm{M}}+\overline{\mathrm{K}}+\sum_{i=1}^{N_{e}} \tilde{\eta}_{e_{i}}(p) \overline{\mathrm{K}}_{e_{i}}-q_{D} \overline{\mathrm{E}}\left(M_{\infty}, p\right)\right] \tilde{\overline{\mathrm{q}}}(p)=q_{D} \overline{\mathrm{E}}_{g}\left(M_{\infty}, p\right) \tilde{w}_{g}(p)
$$

The aeroviscoelastic system (34) has a transcendent dependency on the Laplace variable $p$ through both the unsteady aerodynamics and damping. Nonetheless, the homogeneous solution of Eq. (34) can still be written in the time domain as

$$
\overline{\mathbf{q}}(t)=\sum_{n=1}^{2 N_{m}} c_{n} \mathbf{w}^{(n)} e^{s_{n} t}
$$

where $\mathrm{w}^{(n)}$ and $s_{n}$ are the $n$th aeroviscoelastic eigenvalue and the associated complex eigenvector. These are computed using the non-iterative flutter analysis proposed in Refs. [26, 27]. The method is chosen for the studies of this paper due to its proven robustness and because it is not subject to the convergence issues experienced by iterative approaches [28]. The method solves the flutter equation using a $p-k$ approach for a set of reduced frequencies and uses an inner-loop mode-tracking [29] and a linear interpolation for finding the solutions that verify the condition $k_{n}=\operatorname{Im}\left(p_{n}\right)=\omega_{n} b / U_{\infty}$.

Finally, gust response can be analyzed considering the FRF to the gust input $w_{g}$ given by

$$
\mathrm{H}_{g}(\omega)=q_{D}\left[-\omega^{2} \overline{\mathrm{M}}+\overline{\mathrm{K}}+\sum_{i=1}^{N_{e}} \tilde{\eta}_{e_{i}}(\omega) \overline{\mathrm{K}}_{e_{i}}-q_{D} \overline{\mathrm{E}}\left(M_{\infty}, k\right)\right]^{-1} \overline{\mathrm{E}}_{g}\left(M_{\infty}, k\right)
$$

The transient response of the aeroviscoelastic system to an assigned deterministic gust profile in the time-domain can be obtained by convolving the impulsive response with the assigned gust profile.

\section{TEST CASE}

The test case used in this paper is based on the Body-Freedom-Flutter (BFF) experimental vehicle developed by Lockheed Martin [21]. The configuration was designed to show a bodyfreedom flutter instability due to the coupling of plunge and pitch motions with wing bending and aerodynamics. This instability is typical of tailless configurations and is caused by the exchange of energy between a high-frequency short-period mode and a low-frequency first aeroelastic mode. The BFF also experiences a traditional out-of-plane bending-torsion flutter at higher dynamic pressure. Due to these behaviors, the BFF is a relevant test case for evaluating damping models for passive flutter suppression.

A MSC Nastran aeroelastic model of the BFF was developed in a previous work by the authors [30]. The model was built based on published information on the BFF properties, natural vibration frequencies, and mode shapes [22]. The aeroelastic model consists of a shell-type FEM model coupled with a lifting-surface DLM model through surface splines. The FEM and DLM models are shown in Fig. 1 .

The total model mass is $M=5.457 \mathrm{~kg}$. The center of mass lies in the plane of symmetry at $0.658 \mathrm{~m}$ from of the nose. The roll, pitch, and yaw moments of inertia with respect to the center of mass are equal to $1.14 \mathrm{~kg} \cdot \mathrm{m}^{2}, 0.23 \mathrm{~kg} \cdot \mathrm{m}^{2}$, and $1.37 \mathrm{~kg} \cdot \mathrm{m}^{2}$, respectively, while the products of inertial are practically zero. The frequencies and classifications of the first six free-free natural 

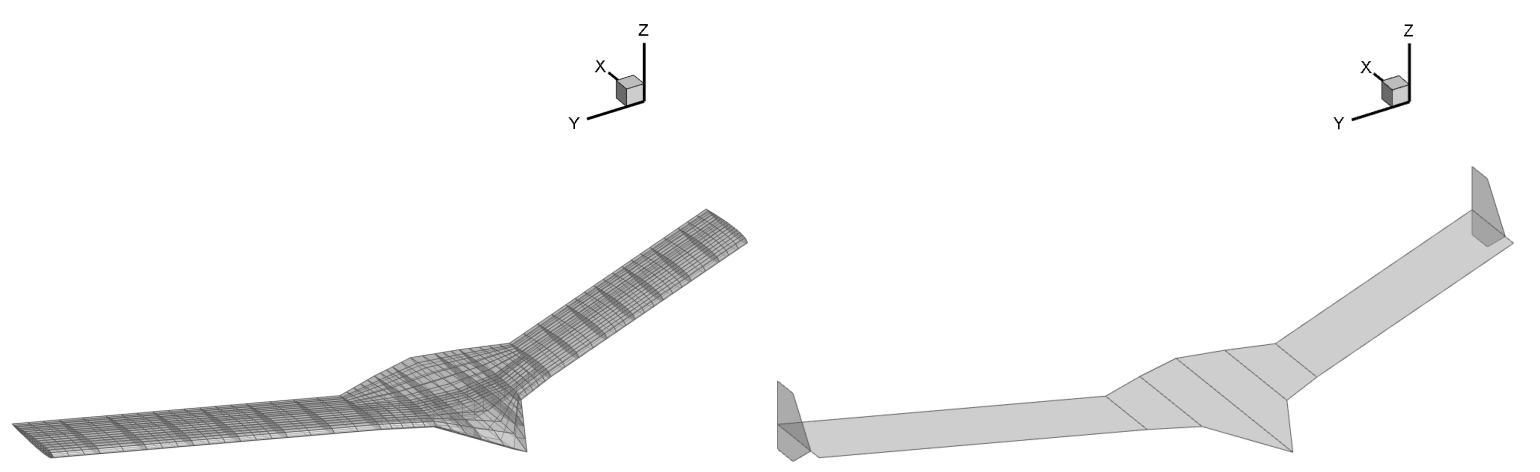

(a) Shell-type FEM model

(b) Lifting-surface DLM model

Figure 1: BFF structural and aerodynamic models [30].

Table 1: BFF free-free natural vibration frequencies [30].

\begin{tabular}{ccc}
\hline Mode \# & Mode type & Frequency $(\mathrm{Hz})$ \\
\hline 1 & Sym out-of-plane bending & 5.83 \\
2 & Asym out-of-plane bending & 8.83 \\
3 & In-plane bending & 13.45 \\
4 & Asym torsion & 19.82 \\
5 & Sym torsion & 20.10 \\
6 & Sym out-of-plane bending & 23.73 \\
\hline
\end{tabular}

vibration modes are reported in Table 1, whereas the corresponding mode shapes are depicted in Fig. 2. The quality of the developed aeroelastic model was assessed in Ref. [30]. Results from a fully coupled flight dynamic and aeroelastic stability analysis were found to agree with published numerical results [22] and flight-test data [21].

\section{RESULTS}

The viscous, hysteretic, and Biot damping models are implemented into the aeroviscoelastic model of the BFF for analyzing flutter and gust response using Eqs. (34) and (36). Prior to this step, the stability of undamped system is studied by assuming $\tilde{\eta}_{e_{i}}=0\left(i=1, \ldots, N_{e}\right)$ and considering steady rectilinear flight at $U_{\infty}=15 \rightarrow 30 \mathrm{~m} / \mathrm{s}$ and $M_{\infty}=0$. These are typical flight conditions for the configuration [21,22]. Rigid-body degrees of freedom are included in the analysis.

The root locus for the first 12 modes is shown in Fig. 3. Aeroelastic modes are labeled according to the structural mode they originate from. The star symbol identifies the body-freedom flutter point that occurs at flight speed $U_{F}=19.27 \mathrm{~m} / \mathrm{s}$ and is associated to an angular frequency $\omega_{F}=$ $26.9 \mathrm{rad} / \mathrm{s}$. The main components of the flutter eigenvector $\mathrm{w}_{F}$ are shown in Table 2 while other components are at least one order of magnitude smaller. As expected for this configuration, the flutter mode involves the symmetric rigid-body motion parameters (plunge and pitch) and the first symmetric out-of-plane bending and torsion structural modes, with a higher participation from bending. 

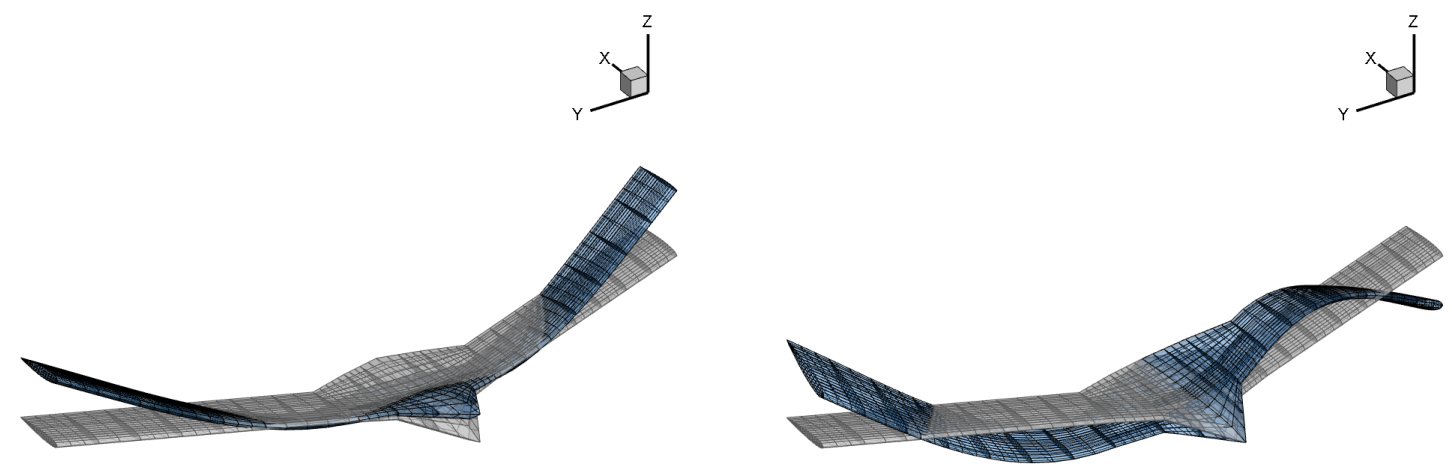

(a) Mode \#1 (sym out-of-plane bending)

(b) Mode \#2 (asym out-of-plane bending)
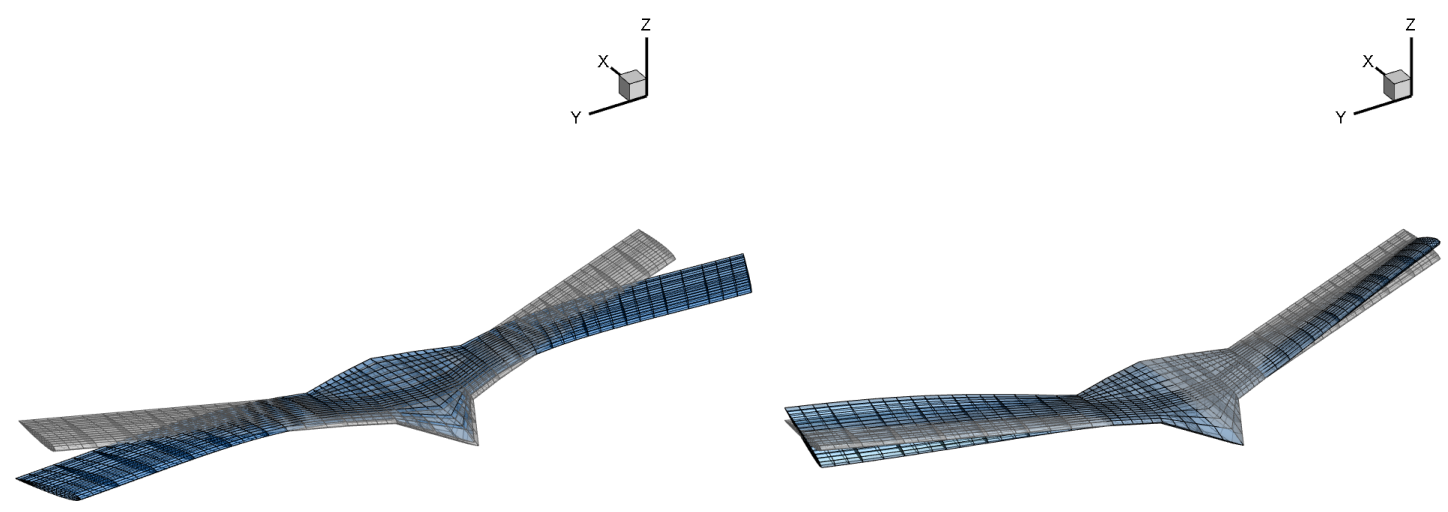

(c) Mode \#3 (in-plane bending)

(d) Mode \#4 (asym torsion)
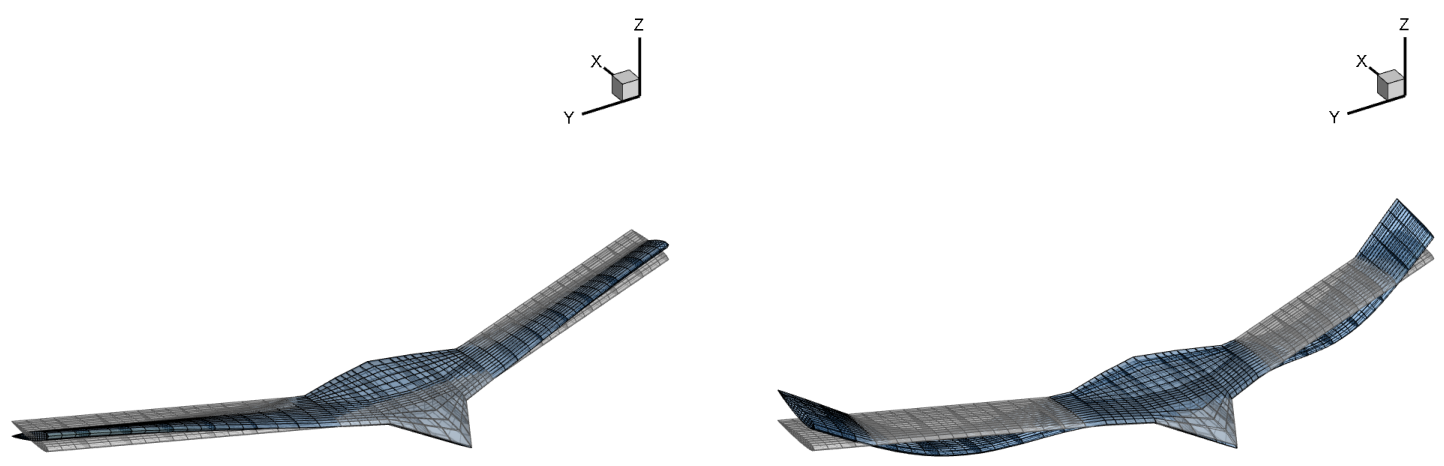

(e) Mode \#5 (sym torsion)

(f) Mode \#6 (sym out-of-plane bending)

Figure 2: BFF free-free natural vibration modes [30]. 


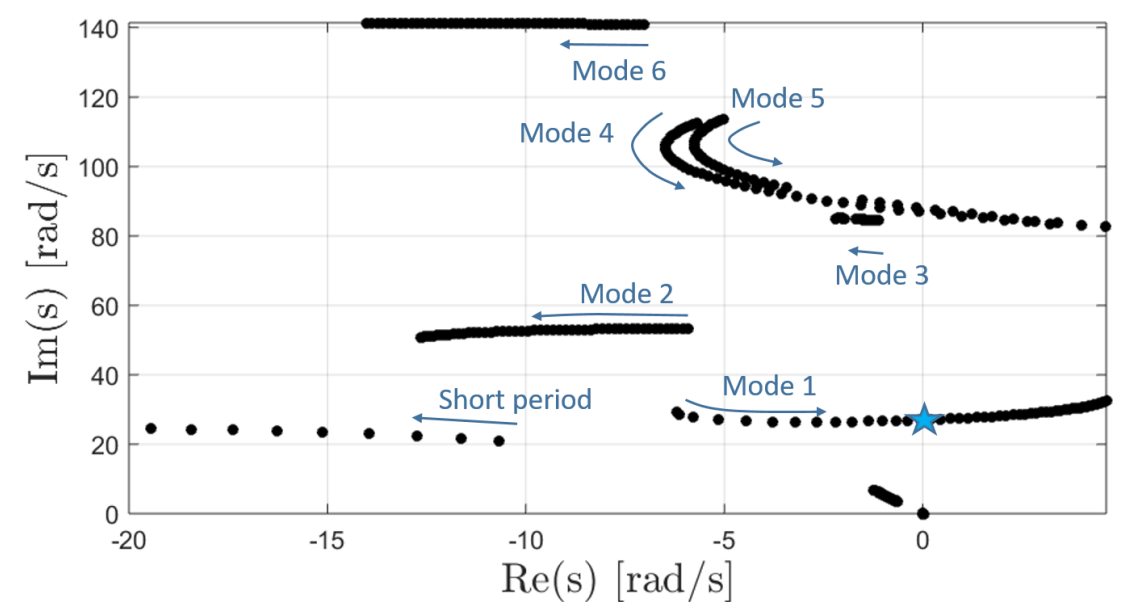

Figure 3: BFF root locus in the absence of damping $\left(U_{\infty}=15 \rightarrow 30 \mathrm{~m} / \mathrm{s}\right.$ and $M_{\infty}=0$ at sea level). Star symbol denotes the flutter point $\left(U_{F}=19.27 \mathrm{~m} / \mathrm{s}\right.$ and $\left.\omega_{F}=26.9 \mathrm{rad} / \mathrm{s}\right)$.

Table 2: BFF flutter mode components associated to plunge $\left(\bar{q}_{\text {plunge }}\right)$, pitch $\left(\bar{q}_{\text {pitch }}\right)$, first symmetric out-of-plane bending mode $\left(\bar{q}_{1}\right)$, and first symmetric torsion mode $\left(\bar{q}_{5}\right)$.

\begin{tabular}{cc}
\hline Generalized coordinate & Component \\
\hline $\bar{q}_{\text {plunge }}$ & $-0.133-\mathrm{j} 0.087$ \\
$\bar{q}_{\text {pitch }}$ & $-0.236-\mathrm{j} 0.128$ \\
$\bar{q}_{1}$ & 0.8436 \\
$\bar{q}_{5}$ & $-0.337-\mathrm{j} 0.026$ \\
\hline
\end{tabular}

For implementing damping into the aeroelastic system, the top wing skin is subdivided into three patches as shown Fig. 4(a). The spatial contribution to the dissipation index $\mathcal{F}_{\mathcal{B}}$ is computed using Eq. (25) for each patch individually considering the flutter mode associated to the critical eigenvector for identifying the most effective patch for suppressing flutter. Figure 4(b) shows that patch \#1 leads to the highest dissipation, while contributions from the other patches are slight. This result is expected because the body-freedom flutter mode involves the first wing symmetric out-of-plane bending and torsion deformations as shown in Table 2. Based on this consideration, damping properties are associated only to patch \#1 for the following analyses.

Having identified the most effective patch, the viscous, hysteretic, and Biot damping models are implemented by assigning the parameters of the Biot model and evaluating the parameters for the hysteretic and damping models such that the three descriptions dissipate the same energy at the flutter frequency, that is

$$
\mathcal{E}_{D_{V}}\left(\omega_{F}\right)=\mathcal{E}_{D_{H}}\left(\omega_{F}\right)=\mathcal{E}_{D_{B}}\left(\omega_{F}\right)
$$

This choice is based on the observation that the Biot model approximates the behavior of viscoelastic materials as a function of frequency more closely with respect to the viscous and hysteretic damping models. It highlights the impact of using a more approximate damping description at frequencies that are different from the equivalence frequency $\omega_{F}$. The Biot model parameters are chosen as $\bar{\eta}_{B}=0.25$ and $\varepsilon=10 \mathrm{rad} / \mathrm{s}$ and are assumed as constant within patch \#1. Using Eq. (37) and assuming $\bar{\omega}=\omega_{F}=26.90 \mathrm{rad} / \mathrm{s}$, the viscous damping model parameter is evaluated as $\zeta=10$ while the hysteretic damping model parameter is evaluated as $\bar{\eta}_{H}=0.20$. The function $\mathcal{F}_{\eta}$ in Eq. (24) for these parameters is shown in Fig. 5. Results highlight that the equivalence of dissipation is ensured only at the flutter frequency. For lower frequencies the vis- 


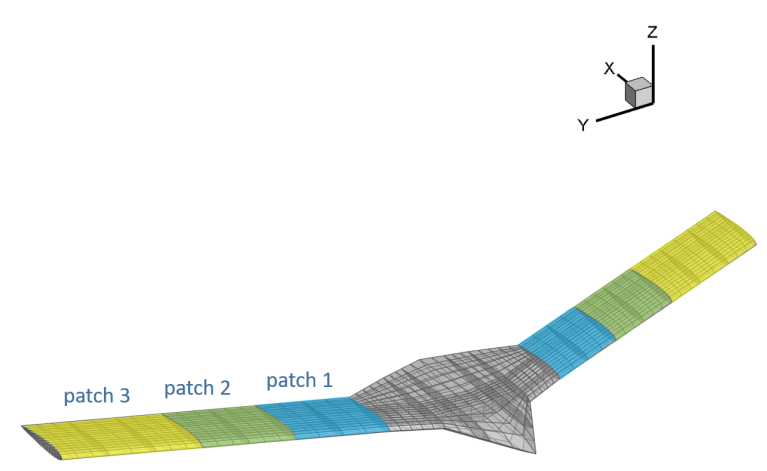

(a) Wing skin patches layout

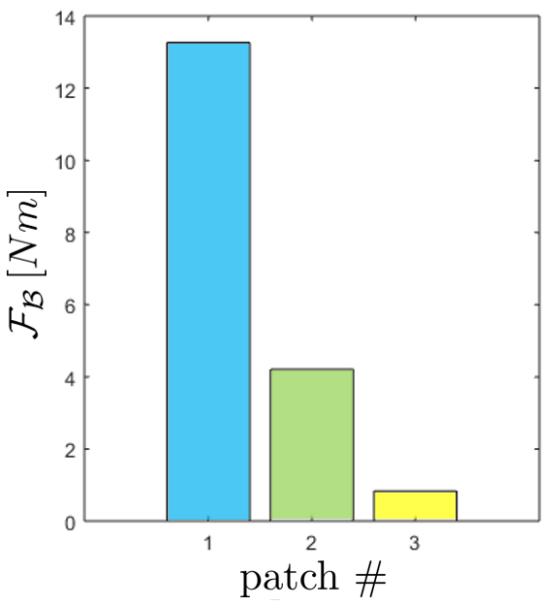

(b) $\mathcal{F}_{\mathcal{B}}$

Figure 4: Contribution to $\mathcal{F}_{\mathcal{B}}$ for the flutter mode in Table 2 from the wing skin patches in Fig. 4(a)

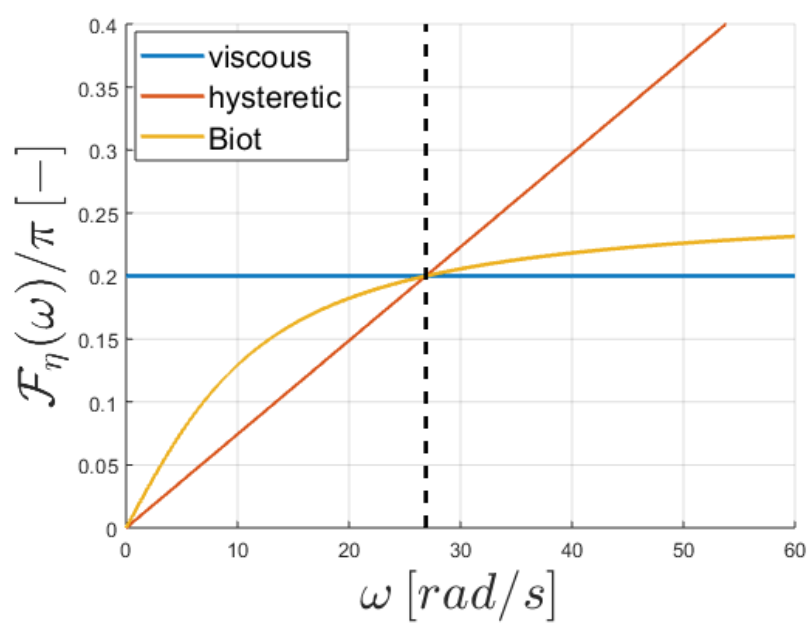

Figure 5: Frequency component of the dissipation metric $\mathcal{F}_{\eta}(\omega) / \pi$ for the viscous, hysteretic, and Biot damping models for energy dissipation equivalence enforced at the body-freedom flutter frequency.

cous model underestimates damping compared to the Biot model whereas the hysteretic model overestimates damping. The opposite occurs for frequencies higher than the flutter frequency.

The root locus for the damped system is shown in Fig. 6 for the same parameters as for Fig. 3 , Figure 7 shows the variation of damping $g=\arctan \left(\operatorname{Re}\left(s_{n}\right) / \operatorname{Im}\left(s_{n}\right)\right)$ with flight speed where flutter points are marked by vertical lines. The viscous and hysteretic damping models give the same slight increase in the body-freedom flutter speed from $U_{F}=19.27 \mathrm{~m} / \mathrm{s}$ (undamped case) to $19.36 \mathrm{~m} / \mathrm{s}$. A higher flutter speed equal to $19.69 \mathrm{~m} / \mathrm{s}$ is obtained with the Biot model due to the effect on the system stiffness in addition to damping (see Eq. (18)). The viscous model highly damps out the second instability (traditional bending-torsion flutter) that is observed for this configuration because the model parameters are obtained by enforcing the equivalence (37) at the frequency of the body-freedom mode. In contrast, the hysteretic damping model gives a lower damping on this second instability compared to the Biot model. These results can be motivated by the variation of $\mathcal{F}_{\eta}(24)$ with frequency shown in Fig. 5 and highlight the limitations of damping models that approximate the viscoelastic behavior given by the Biot model. The three damping formulations achieve similar effects only at one chosen frequency, 


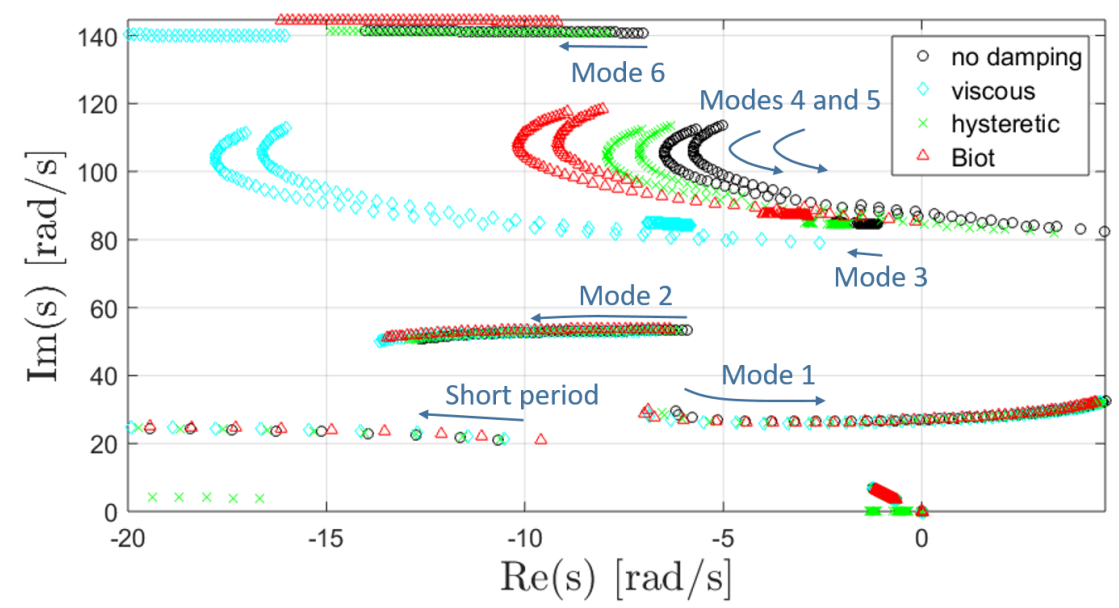

Figure 6: Root loci obtained by different damping models.

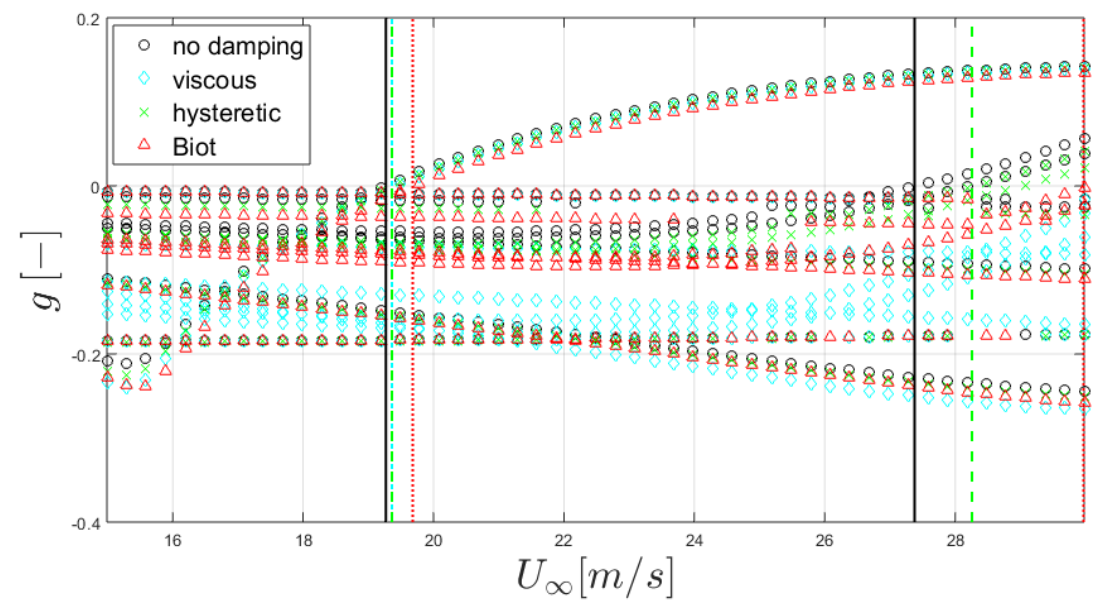

Figure 7: Damping as a function of speed as provided by different damping models.

whereas their effect is different at lower or higher frequencies. Additionally, note that the hysteretic model gives spurious poles at low frequency (see Fig. 6) due to the fact that the real and imaginary parts of the complex stiffness matrix do not form a Hilbert pair.

For completeness, gust response is also analyzed in the frequency domain using the FRF in Eq. (11). The magnitude of the FRFs for plunge $\left(\bar{q}_{\text {plunge }}\right)$, pitch $\left(\bar{q}_{\text {pitch }}\right)$, first symmetric out-ofplane bending mode $\left(\bar{q}_{1}\right)$, and first symmetric torsion mode $\left(\bar{q}_{5}\right)$ are shown in Fig. 8 . Results are for $U_{\infty}=0.90 U_{F}=17.4 \mathrm{~m} / \mathrm{s}$. As observed from the stability analysis, the Biot model gives a lower FRF amplitude compared to the other two models at the first peak and it introduces a frequency shift that is more apparent for the second, higher frequency peak due to the effect on the system stiffness. The viscous damping model highly attenuates the second peak, while the hysteretic model has only a slight impact.

The transient responses for the DOFs in Fig. 8 are shown in Fig. 9 . Results are obtained by considering a deterministic vertical 1-cosine gust with maximum amplitude $w_{g_{\max }}=1 \mathrm{~m} / \mathrm{s}$ and a characteristic length $L_{g}=12 b$. Results are in line with the frequency-domain stability and response analyses. 


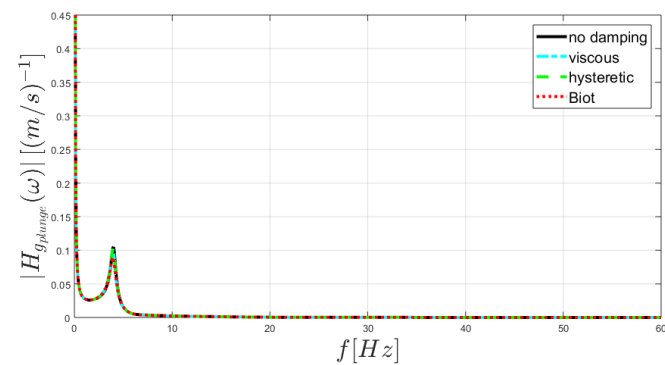

(a) Heave

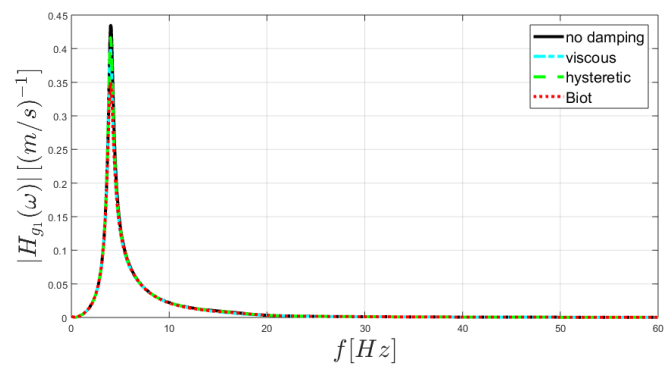

(c) Mode \#1

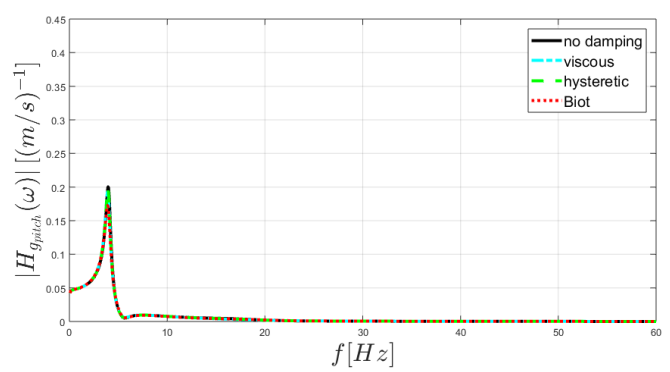

(b) Pitch

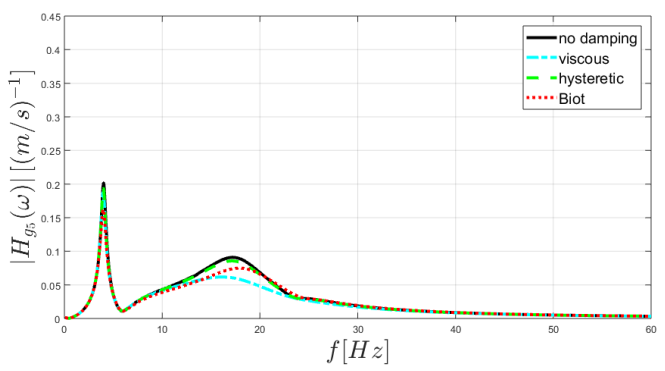

(d) Mode \#5

Figure 8: BFF frequency-domain FRF for a gust load and for the for plunge $\left(\bar{q}_{\text {plunge }}\right)$, pitch $\left(\bar{q}_{\text {pitch }}\right)$, first symmetric out-of-plane bending mode $\left(\bar{q}_{1}\right)$, and first symmetric torsion mode $\left(\bar{q}_{5}\right)$.

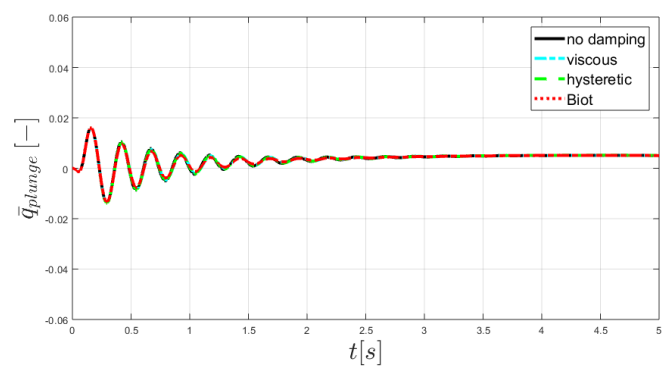

(a) Heave

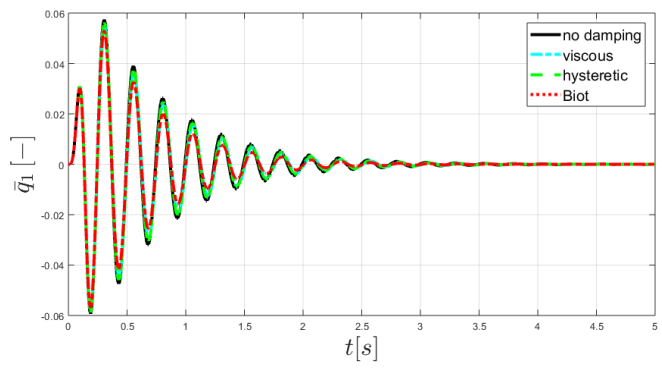

(c) Mode \#1

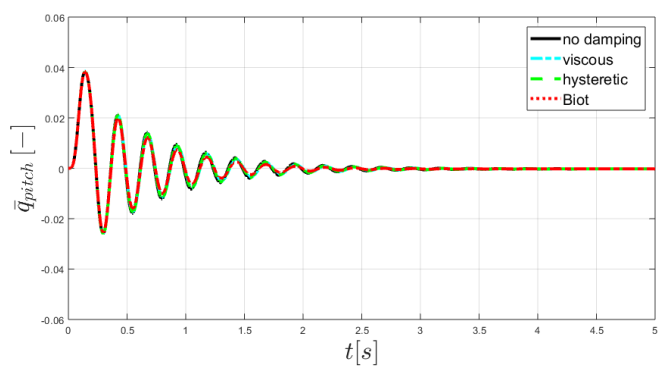

(b) Pitch

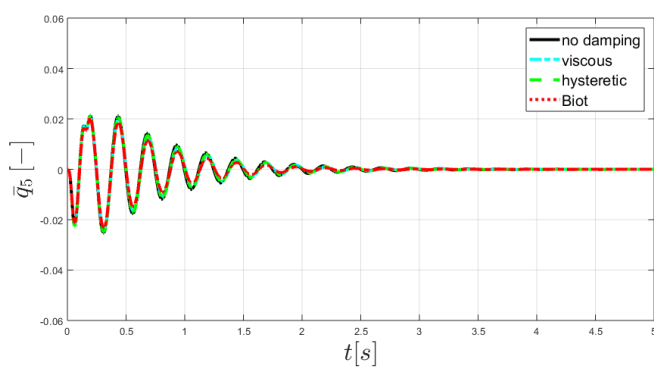

(d) Mode \#5

Figure 9: BFF time-domain response to a vertical 1-cosine gust load $\left(w_{g_{\max }}=1 \mathrm{~m} / \mathrm{s}\right.$ and $\left.L_{g}=12 b\right)$ and for the for plunge $\left(\bar{q}_{\text {plunge }}\right)$, pitch $\left(\bar{q}_{\text {pitch }}\right)$, first symmetric out-of-plane bending mode $\left(\bar{q}_{1}\right)$, and first symmetric torsion mode $\left(\bar{q}_{5}\right)$. 


\section{CONCLUDING REMARKS}

This paper compared the modeling capabilities of the viscous, hysteretic, and generalized Biot damping models as applied to flutter suppression and gust response alleviation studies. To compare the models, a dissipation metric was introduced that quantifies the energy dissipated over one oscillation cycle of a fully developed mono-frequency motion with a arbitrarily assigned displacement shape. The BFF testbed developed by Lockheed Martin was considered as the test case for the studies. The damping models were implemented into the FEM aeroviscoelastic model of the BFF and their parameters were chosen to achieve the same dissipation at the bodyfreedom flutter frequency of the undamped system. Damping properties were associated to a specific top wing skin patch chosen by means of a sensitivity study on the damping effectiveness of different patches for the selected flutter mode.

Frequency-domain stability and response analyses and transient response analyses showed that the three formulations give similar results at the body-freedom flutter frequency, as expected because of the choice of the model parameters, whereas results differ at lower and higher frequencies. Moreover, the Biot model introduces a shift in the system frequencies due to the contribution to the real part of the complex stiffness matrix arising from the Hilbert correction that is not present when using the viscous or the hysteretic model. These results highlights that damping descriptions that approximate viscoelastic behavior such as the viscous and hysteretic models require special care in the choice of their typical parameters based on available material data. In fact, results are in agreement with a viscoelastic description only a one selected frequency whereas damping effects are overestimated or underestimated at other frequencies.

\section{REFERENCES}

[1] Boltzmann, S. (1874). Sitz ber. akad. wiss. wien, math. naturw. kl. Pogg. Ann. Phys., 70(275).

[2] Coleman, B. D. and Noll, W. (1961). Foundations of linear viscoelasticity. Review of Modern Physics, 2. doi:10.1103/RevModPhys.33.239.

[3] Coleman, B. D. and Noll, W. (1965). The nonlinear field theories of mechanics. Encyclopedia of Physics, III(3).

[4] Lee, E. (1960). Viscoelasticity. New York, USA: J. T. Bergen (Academic Press, Inc.).

[5] Gross, B. (1953). Mathematical Structure of the Theories of Viscoelasticity. Paris, 1953: Hermann R Cie.

[6] Fichera, G. (1982). Sul principio della memoria evanescente. Rend. Sem. Mat. Univ. Padova, 68, 245-259.

[7] Fichera, G. (1980). Analytic problems of materials with memory. Proceedings of IUTAM Conference.

[8] Adhikari, S. (2014). Structural Dynamic Analysis with Generalized Damping Models: Analysis. London, UK: Wiley-ISTE.

[9] Mastroddi, F., Martarelli, F., Eugeni, M., et al. (2019). Time- and frequency-domain linear viscoelastic modeling of highly damped aerospace structures. Mechanical Systems and Signal Processing, 122, 42-45. doi:10.1016/j.ymssp.2018.12.023. 
[10] Mastroddi, F., Eugeni, M., , et al. (2017). On the modal diagonalization of viscoelastic mechanical systems. Mechanical Systems and Signal Processing, 96, 159-175. doi:10. 1016/j.ymssp.2017.04.009.

[11] Riso, C., Fransen, S., Mastroddi, F., et al. (2018). Experimental validation of solid rocket motor damping models. CEAS Space Journal, 10(2), 213-23. doi:10.1007/ s12567-017-0191-3.

[12] Anon (2013). Dynamic Analysis User's Guide. MSC Software Corporation.

[13] Bagley, L., R. and Torvik, J., P. (1983). A theoretical basis for the application of fractional calculus to viscoelasticity. Journal of Rheology, 27, 201-210. doi:10.1122/1.549724.

[14] Torvik, J., P. and Bagley, L., R. (1984). On the appearance of the fractional derivative in the behavior of real materials. Journal of Applied Mechanics, 51, 294-298. doi:10.1115/ 1.3167615 .

[15] Pritz, T. (1996). Analysis of four parameter fractional derivative model of real solid materials. Journal of Sound and Vibration, 195, 103-115. doi:10.1006/jsvi.1996.0406.

[16] Meza-Fajardo, K. C. and Lai, C. G. (2007). Explicit causal relations between material damping ratio and phase velocity from exact solutions of the dispersion equations of linear viscoelasticity. Geophysical Journal International, 171, 1247-1257. doi: 10.1111/j.1365-246X.2007.03590.

[17] Biot, M. A. (1958). Linear thermodynamics and the mechanics of solids. New York, NY: Proceedings of the Third U.S. National Congress of Applied Mechanics.

[18] Mainardi, F. and Spada, G. (2011). Creep, relaxation and viscosity properties for basic fractional models in rheology. The European Physical Journal, 193, 133-160. doi:10. 1140/epjst/e2011-01387-1.

[19] Eugeni, M., Mastroddi, F., and Dowell, E. (2017). Normal form analysis of a forced aeroelastic plate. Journal of Sound and Vibration, 390, 141-163. doi:10.1016/j.jsv.2016. 12.001 .

[20] Eugeni, M., Dowell, E., and Mastroddi, F. (2014). Post-buckling longterm dynamics of a forced nonlinear beam: A perturbation approach. Journal of Sound and Vibration, 333(9), 2617-2631. doi:10.1016/j.jsv.2013.12.026.

[21] Burnett, E., Atkinson, C., Beranek, J., et al. (2010). NDOF simulation model for flight control development with flight test correlation. Toronto, Ontario, CA: AIAA Modeling and Simulation Technologies Conference. doi:10.2514/6.2010-7780.

[22] Schmidt, D. (2016). Stability augmentation and active flutter suppression of a flexible flying-wing drone. Journal of Guidance, Control, and Dynamics, 39(3), 409-422. doi: 10.2514/1.G001484.

[23] Nashif, A. D., Jones, D. I. G., and Henderson, J. P. Vibration Damping.

[24] Makris, N. (1998). Frequency-independent dissipation and causality. Santorini, Greece: Third International Conference on Computational Stochastic Mechanics. 
[25] Ablowitz, M. J. and Fokas, A. S. (2003). Complex Variables: Introduction and Applications. Cambridge, UK: Cambridge University Press, 2 ed. doi:10.1017/ CBO9780511791246.

[26] Van Zyl, L. (2001). Aeroelastic divergence and aerodynamic lag roots. Journal of Aircraft, 38(3), 586-587. doi:10.2514/2.2806.

[27] Chen, P. (2000). Damping perturbation method for flutter solution: The g-method. AIAA journal, 38(9), 1519-1524. doi:10.2514/2.1171.

[28] Jonsson, E., Mader, C. A., Kennedy, G. J., et al. (2019). Computational modeling of flutter constraint for high-fidelity aerostructural optimization. In 2019 AIAA/ASCE/AHS/ASC Structures, Structural Dynamics, and Materials Conference. San Diego, CA. doi:10.2514/ 6.2019-2354.

[29] Van Zyl, L. (1993). Use of eigenvectors in the solution of the flutter equation. Journal of Aircraft, 30(4), 553-554. doi:10.2514/3.46380.

[30] Saltari, F., Riso, C., De Matteis, G., et al. (2017). Finite-element-based modeling for flight dynamics and aeroelasticity of flexible aircraft. Journal of Aircraft, 54(6), 2350-2366. doi:10.2514/1.C034159.

\section{COPYRIGHT STATEMENT}

The authors confirm that they, and/or their company or organization, hold copyright on all of the original material included in this paper. The authors also confirm that they have obtained permission, from the copyright holder of any third party material included in this paper, to publish it as part of their paper. The authors confirm that they give permission, or have obtained permission from the copyright holder of this paper, for the publication and distribution of this paper as part of the IFASD-2019 proceedings or as individual off-prints from the proceedings. 